Acta Zoológica Mexicana (nueva serie), Volumen 37, 1-10.

https://doi.org/10.21829/azm.2021.3712403

Artículo original

\title{
Actualización de las especies del género Macrodactylus Dejean (Coleoptera: Melolonthinae: Macrodactylini) para el estado de Puebla, México
}

\section{Update of the species of the genus Macrodactylus Dejean (Coleoptera: Melolonthinae: Macrodactylini) for the state of Puebla, Mexico}

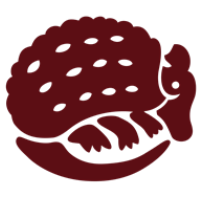

OPEN ACCESS
*Autor corresponsal:

D Agustín Aragón García agustin.aragon@correo.buap.mx

Cita:

Aragón-Sánchez, M., Aragón, G. A., Arce-Pérez, R., Pérez, T. B. C., Cuate-Mozo, V. A., López-Olguín, J.

F. (2021) Actualización de las especies del género Macrodactylus Dejean (Coleoptera: Melolonthinae: Macrodactylini) para el estado de Puebla, México. Acta Zoológica Mexicana (nueva serie), 37, 1-10.

10.21829/azm.2021.3712403 elocation-id: e3712403

Recibido: 25 marzo 2021 Aceptado: 17 agosto 2021 Publicado: 20 agosto 2021

\section{Miguel ARAGóN-SÁNCHEZ, (iD 1*Agustín ARAGón GARCíA, ${ }^{2}$ Roberto ARCE-PÉREZ, ID ${ }^{1}$ BetZabeth CeCILIA PÉREZ TORRES, (iD) ${ }^{3}$ Víctor AlFonzo CUATE-MOZO, (iD 1 Jesús Francisco LóPEZ- OLGUÍN}

${ }^{1}$ Centro de Agroecología, Instituto de Ciencias, Benemérita Universidad Autónoma de Puebla. EcoCampus Valsequillo. Edificio VAL 1, Km 1.7 carretera San Baltazar Tetela, C.P. 72960. San Pedro Zacachimalpa, Puebla, México.

${ }^{2}$ Instituto de Ecología, A. C. Carretera Antigua a Coatepec 351, El Haya, C.P. 91073, Xalapa, Veracruz, México.

${ }^{3}$ Colegio de Ciencias Agropecuarias, Facultad de Agricultura del Valle del Fuerte, Universidad Autónoma de Sinaloa. Calle 16 y avenida Japaraqui, Juan José Ríos, C.P. 81110, Ahome, Sinaloa, México.

Editor responsable: Alfredo Ramírez-Hernández

RESUMEN. El estado de Puebla, México, ocupa el quinto lugar en riqueza de especies del género Macrodactylus con nueve especies. Por medio de la revisión de colecciones científicas y trabajo de campo, se actualizó el número de especies a diez, con Macrodactylus lineatocollis Bates 1887, como nuevo registro estatal. Se presentan mapas con su distribución territorial, que se relacionan con las variables de temperatura media anual y rangos de humedad. Se hacen comentarios biológicos para cada especie y se presenta una clave dicotómica para la identificación de las especies presentes en el estado de Puebla.

Palabras clave: coleópteros; escarabajos mexicanos; insectos plaga; gallina ciega; distribución de escarabajos 
ABSTRACT. The state of Puebla, Mexico, ranks fifth in species richness of the genus Macrodactylus with nine species. Through the revision of scientific collections and field work, the number of species was actualized to ten, with Macrodactylus lineatocollis Bates 1887, as a new state registry. Maps are presented with their territorial distribution, which are related to the variables of mean annual temperature and humidity ranges. Biological comments are made for each species and a dichotomous key is presented for the identification of the species present in the state of Puebla.

Key words: coleopterans; Mexican beetles; pest insects; white grub; beetle's distribution

\section{INTRODUCCIÓN}

El género americano Macrodactylus Dejean (Melolonthinae: Macrodactylini) incluye 122 especies distribuidas desde el sureste de Canadá hasta el centro sur de Argentina y Chile. En México se han registrado 28 especies, con la mayor riqueza en los estados de México (12), Oaxaca (12), Chiapas (11), Michoacán (11), Puebla (9) y Durango (8) (Arce-Pérez \& Morón, 2005; 2011; 2014; 2018; 2020; Evans, 2003; Evans \& Smith, 2005).

En México se les conoce como "frailecillos", "taches" o "escarabajos de las rosas" por el patrón pardo oscuro a grisáceo que presenta su exoesqueleto; sin embargo, las características más sobresalientes son el largo y lo esbelto de sus cuerpos (Figs. 1-6), así como la longitud de sus patas (de allí la denominación de Macrodactylus = patas grandes). Estos escarabajos con frecuencia son citados como plagas de cultivos agrícolas y forestales; sus larvas, conocidas como "gallinas ciegas" causan daños en las raíces de cultivos como el maíz (Zea mays L.), trigo (Triticum spp.) y centeno (Secale cereale (L.) M. Bieb) de la familia Poaceae; el fríjol (Phaseolus vulgaris L.), haba (Vicia faba L.) y alfalfa (Medicago sativa L.) de la familia Fabaceae; nabo (Brassica rapa L.) (Brassicaceae) y pastos forrajeros y de ornato, mientras que los adultos se alimentan de follaje en crecimiento, flores, frutos, granos de polen y secreciones de savia dulce de los cultivos mencionados, así como de árboles silvestres y cultivados como el pino (Pinus spp.) (Pinaceae), trueno (Ligustrum spp.) (Oleaceae), sauce (Salix spp.) (Salicaceae), pirul (Schinus molle L.) (Anacardiaceae), tepozán (Buddleja cordata Kunth) (Scrophulariaceae), tejocote (Crataegus spp.) (Rosaceae), capulín (Prunus salicifolia Kunth) (Rosaceae), guayaba (Psidium spp.) (Myrtaceae), pera (Pyrus spp.) (Rosaceae), durazno (Prunus persica (L.) Batsch) (Rosaceae), manzano (Malus domestica Borkh) (Rosaceae), ciruelo (Prunus domestica L.) (Rosaceae), vid (Vitis spp) (Vitaceae), aguacate (Persea spp.) (Lauraceae), níspero (Eriobotrya japonica (Thunb) Lindl) (Rosaceae), moras (Morus spp.) (Rosaceae), zarzamora (Rubus fructicocus L.) (Rosaceae), frambuesa (Rubus idaeus L.) (Rosaceae), café (Coffea spp.) (Rubiaceae) y muchos otros, en los cuales pueden causar daños de importancia, siendo fácil observar cientos de ellos en una sola planta (Arce-Pérez \& Morón, 2000; 2009; 2010).

El objetivo de este trabajo es actualizar el registro de las especies del género Macrodactylus para el estado de Puebla con un nuevo registro estatal, generar mapas de presencia estatal con los datos de distribución y preferencias ecológicas y, con ayuda de mapas de temperatura media anual y rango de humedad, analizar la relación de la distribución del género con estas variables 
climáticas, listar las principales plantas hospederas y proporcionar una clave dicotómica de las especies para su fácil reconocimiento.
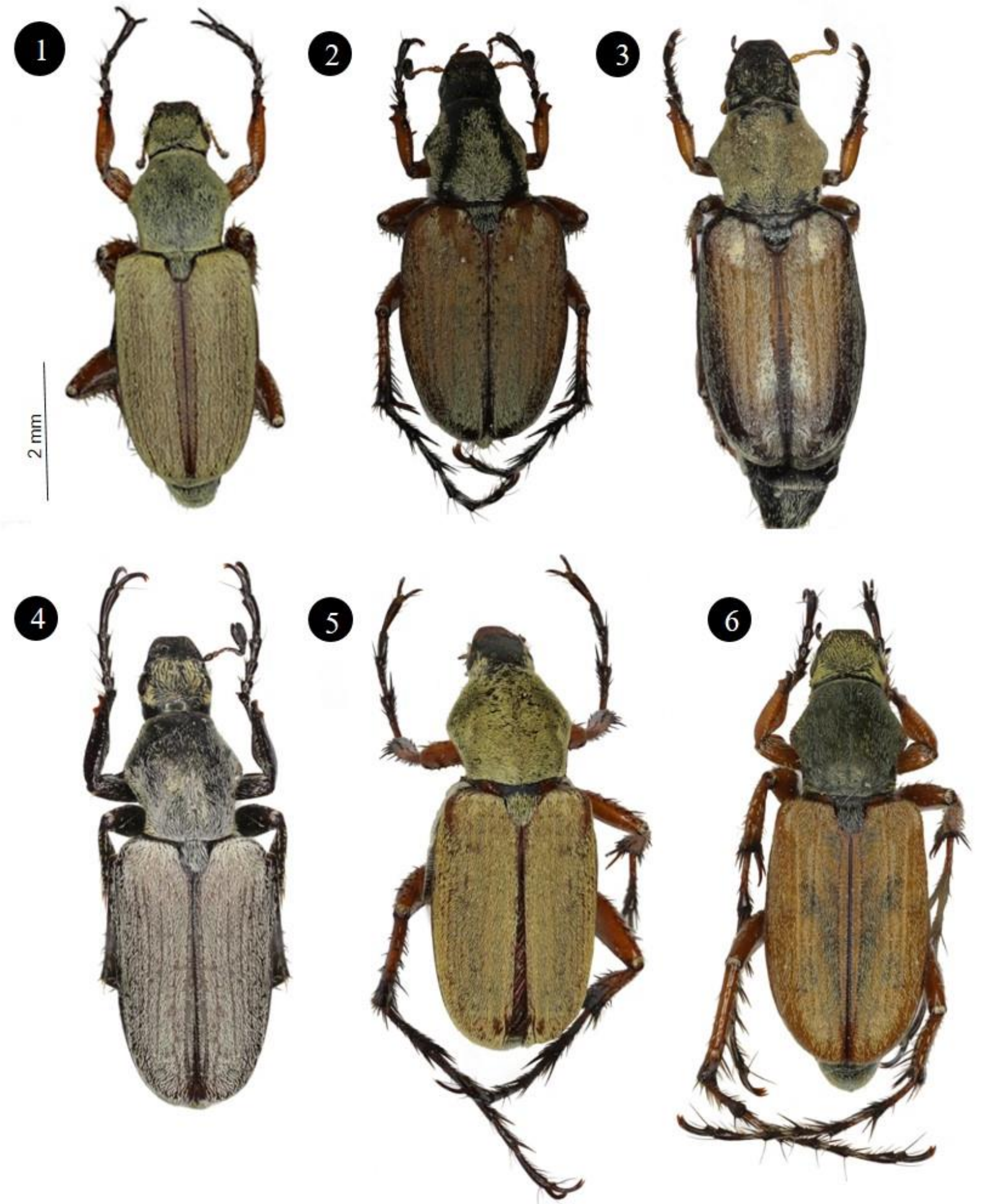

Figuras 1-6. Vista dorsal de Macrodactylus sp. 1) M. fulvescens, 2) M. lineatocollis, 3) M. mexicanus, 4) M. nigripes, 5) M. ocreatus, 6) M. rufescens. 


\section{MATERIALES Y MÉTODOS}

Los registros del género Macrodactylus del estado de Puebla derivan de la revisión de la Colección Entomológica "Miguel Ángel Morón Ríos" del Centro de Agroecología (CENAGRO) del Instituto de Ciencias, de la Benemérita Universidad Autónoma de Puebla (BUAP) y la Colección Entomológica (IEXA) del Instituto de Ecología A.C., Xalapa, Veracruz; además, algunos ejemplares provenían de recolectas esporádicas sobre vegetación realizadas entre mayo y septiembre del 2015 al 2019 en la mayoría de los municipios que conforman el estado de Puebla, principalmente en zonas agrícolas. Los ejemplares recolectados se sacrificaron con vapores de acetato de etilo. Se montó un $10 \%$ de las morfoespecies en alfileres entomológicos y el resto se fijó con alcohol al 70\%. Los ejemplares se determinaron taxonómicamente de acuerdo con las claves propuestas por ArcePérez y Morón (2000) y Arce-Pérez (2008). Los ejemplares fueron depositados en la colección entomológica "Miguel Ángel Morón Ríos" del CENAGRO del Instituto de Ciencias de la BUAP.

Con los datos de presencia de las especies se generaron mapas de distribución del género Macrodactylus a nivel municipal. Asimismo, se analizó la relación entre las ocurrencias de las especies con la temperatura media anual y rangos de humedad (García, 1990), sobreponiendo a modo de capas los registros de las especies sobre los mapas de temperatura y humedad. Los mapas de distribución del género y la influencia de la temperatura media anual y rangos de humedad se construyeron con el programa gvSIG Desktop 2.5 (GNU GPLv3 Java, Valencia, España).

Una vez identificado y determinado todo el material y, de acuerdo con sus características morfológicas, se elaboró una clave dicotómica para identificar adultos del género Macrodactylus del estado de Puebla.

\section{RESULTADOS Y DISCUSIÓN}

Dos décadas atrás se había registrado en el estado de Puebla nueve especies de Macrodactylus (Arce-Pérez \& Morón, 2000). Téllez-Carmona et al. (2018) menciona la presencia de Macrodactylus lapuscatus Bates asociada al cultivo de chía Salvia hispánica L. (Lamiaceae) en la localidad de Tochimilco, Municipio de Tochimilco; sin embargo, al realizar la revisión taxonómica de este material se identificó y reubicó como Macrodactylus lineatocollis Bates, 1887. En consecuencia, esta especie constituye un nuevo registro para el estado de Puebla incrementando su diversidad estatal a 10 especies, que representan el $35.7 \%$ del total registradas para México, clasificadas en los grupos Lineatus, Dimidiatus y Subspinosus de acuerdo con Arce-Pérez y Morón (2000) (Cuadro 1).

Respecto a la distribución de las especies de Macrodactylus con relación a la temperatura media anual, en el mapa se distinguen los siguientes patrones de distribución de especies: 1) Los mayores registros de ocurrencia de las especies de este género se ubican en los municipios con temperaturas de los $12^{\circ} \mathrm{C}$ a $22^{\circ} \mathrm{C}$, temperaturas consideradas dentro de los climas semifríos, templados y semicálidos; 2 ) No se observan ocurrencias en las temperaturas cálidas, superiores de $22^{\circ} \mathrm{C}$ a $26^{\circ} \mathrm{C}$, lo que corresponde a los municipios de la mixteca poblana; 3) No hay registros de especies en las temperaturas frías inferiores a una temperatura media anual de $5^{\circ} \mathrm{C}$ (Fig. 8). 
En el mapa de rangos de humedad se distingue que las especies de este género prefieren la zona con clima húmedo y subhúmedo, no encontrándose en lugares con un porcentaje de lluvia inferior al 5\% (W0). En el caso de la colecta de Macrodactylus ocreatus Bates, 1887, en la zona de Zapotitlán Salinas, la especie se distribuye hacia la parte más húmeda del municipio, que corresponde a San Pedro Atzumba, Zapotitlán Salinas y San Juan Acatitlán, Caltepec, donde el clima semiárido es templado con temperatura media anual entre $12^{\circ} \mathrm{C}$ y $18^{\circ} \mathrm{C}(\mathrm{CIBCEC}, 2003)$ (Fig. 9).

Las especies con distribución más amplia en el estado son Macrodactylus mexicanus Burmeister, 1845 y Macrodactylus nigripes Bates, 1887, ambas especies ocupan las mayores ocurrencias en los municipios de la porción centro y sierra norte de Puebla, además, a estas especies se le ha encontrado en diferentes zonas agrícolas en donde causan daños importantes para la agricultura Poblana, al igual que en diferentes ecosistemas agrícolas de América Central (Coto \& Saunders, 2004; Saunders et al., 1983).

Cuadro1. Especies de Macrodactylus presentes en el estado de Puebla.

\begin{tabular}{ll}
\hline Grupo & Especie \\
\hline \multirow{3}{*}{ I, Lineatus } & M. fulvescens Bates, 1887 \\
& M. ocreatus Bates, 1887 \\
& M. lineatus Chevrolat, 1834 \\
\hline II, Dimidiatus & M. dimidiatus Guerin-Menedille, 1844 \\
\hline & M. rufescens Bates, 1887 \\
M. mexicanus Burmeister, 1845 \\
IV, Subspinosus \\
M. nigripes Bates, 1887 \\
M. infuscatus Bates, 1887 \\
M. lineatocollis Bates, 1887 \\
M. impresus Bates, 1887
\end{tabular}

\section{Comentarios sobre las especies de Macrodactylus presentes en el estado de Puebla}

Los ejemplares utilizados para realizar los comentarios de las especies, así como para la clave de identificación se encuentran depositados en la Colección Entomológica "Miguel Ángel Morón Ríos" del Instituto de Ciencias de la Benemérita Universidad Autónoma de Puebla (BUAP).

\section{Grupo I Lineatus}

Macrodactylus fulvescens Bates, 1887. Los adultos fueron recolectados entre junio y julio sobre plantas de Amaranthus hypochondriacus (Amaranthaceae) y Z. mays, en los municipios de Atlixco, Huaquechula (en la localidad de Champusco), Huauchinango, Juan Galindo (en la localidad de Necaxa) y Tochimilco. Datos adicionales en Arce-Pérez y Morón (2000) (Fig. 1, Fig. 10).

Macrodactylus lineatus Chevrolat, 1834. Esta especie se encontró durante el mes de junio en el municipio de Xicotepec, asociada a cultivo de P. vulgaris. Esta recolecta concuerda con lo 
propuesto por Arce-Pérez y Morón (2000), donde mencionan que esta es una especie con distribución principalmente sobre la vertiente oriental del país. En el estado de Puebla sólo se recolectó en las colindancias con el estado de Veracruz. Datos adicionales en Arce-Pérez y Morón (2000) (Fig. 10).

Macrodactylus ocreatus Bates, 1887. Los adultos de esta especie (Fig. 5) se recolectaron durante los meses de mayo y junio alimentándose del árbol de Schinus sp. (Anacardiaceae), así como de la floración de Acacia sp. (Leguminosae) en la zona amarantera de los municipios de Zapotitlán Salinas y Caltepec. Las larvas se encontraron asociadas a la raíz de las plantas de amaranto $A$. hypochondriacus con una población de hasta 12 larvas por mata de amaranto. Datos adicionales en Arce-Pérez y Morón (2000) (Fig. 10).
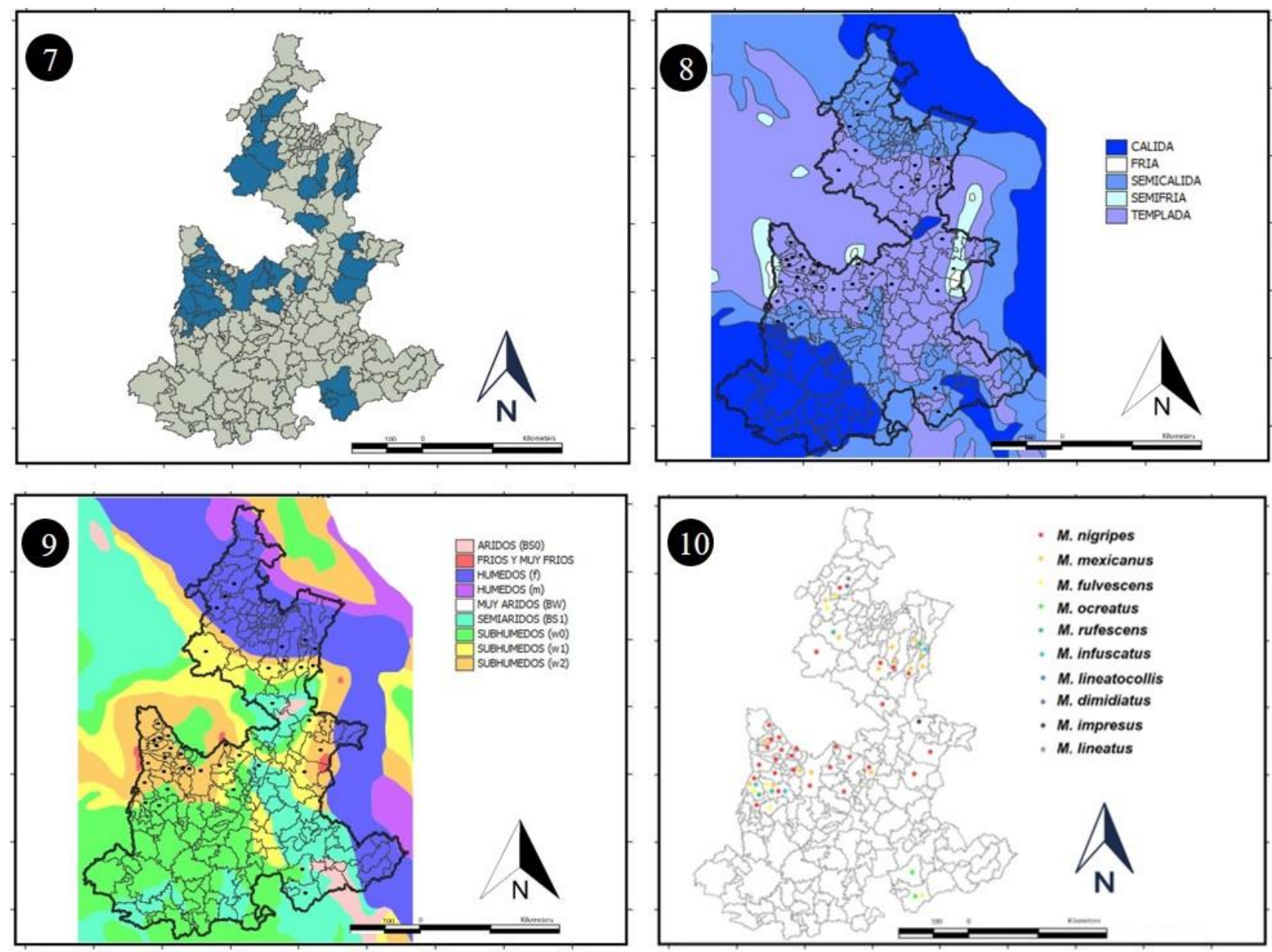

Figuras 7-10. Mapas de distribución del género Macrodactylus en el estado de Puebla. 7) Distribución por presencia en municipios, 8) Temperatura Media Anual del estado de Puebla, indicando presencia del género, 9) Rangos de Humedad para el estado de Puebla, indicando presencia del género, 10) Registros de presencia de las especies del género Macrodactylus en el estado de Puebla registrados en las colecciones entomológicas CENAGRO, BUAP y en IEXA. 


\section{Grupo II Dimidiatus}

Macrodactylus dimidiatus Guerin-Menedille, 1844. Los adultos de esta especie no aparecieron durante los muestreos, pero se reportan en la localidad de Villa Juárez perteneciente al municipio de Xicotepec, principalmente en plantas de $Z$. mays, en los meses de mayo, junio y julio. Datos adicionales en Arce-Pérez y Morón (2000) (Fig. 10).

\section{Grupo IV Subspinosus}

Macrodactylus impresus Bates, 1887. Los adultos de esta especie no aparecieron durante los muestreos, pero se reporta en el municipio de Guadalupe Victoria (Arce-Pérez \& Morón, 2000) sobre plantas de $S$. cereale, follaje de Pinus y algunos pastos no identificados, principalmente entre los meses de abril a junio. Datos adicionales en Arce-Pérez y Morón (2000) (Fig. 10).

Macrodactylus infuscatus Bates, 1887. Los adultos de esta especie se recolectaron en frutos de Pyrus cumunis L. (Rosaceae), y en Salvia hispanica L. (Lamiaceae) en los meses de mayo y junio, en los municipios de Teziutlán y Tochimilco, además de registros publicados en el municipio de Atlixco. Datos adicionales en Arce-Pérez y Morón (2000) (Fig. 10).

Macrodactylus lineatocollis Bates, 1887. Los adultos de esta especie se recolectaron alimentándose del follaje y frutos de E. japonica, mientras que las larvas se encontraron en el pasto Pennisetum clandestinum Hochst ex Chiov (Poaceae). Esta especie se distribuye solamente en el municipio de Atlixco y representa un nuevo registro para el estado de Puebla. Datos adicionales en Arce-Pérez y Morón (2000); Arce-Pérez y Asiain (2008) (Figs. 2, 10).

Macrodactylus mexicanus Burmeister, 1845. Los adultos de esta especie se recolectaron sobre plantas de Z. mays (Poaceae), P. vulgaris y $V$. faba, en los municipios de Acajete, Acatzingo, Amozoc, Atlixco, Atzitzihacán, Calpan, Chinautla, Chiautzingo Chignahuapán, Chalchicomula de Sesma, Huejotzingo, Libres, Juan C. Bonilla, Puebla, San Andrés Cholula, San Felipe Teotlancingo, San Matías Tlalancaleca, San Martín Texmelucan, San Miguel Xoxtla, San Nicolás de los Ranchos, Santiago Zautla, San Pedro Cholula, Santiago Zautla, Tecali de Herrera, Tepatlaxco de Hidalgo, Tianguismanalco, Tlachichuca, Tochimilco, Xicotepec y Zaragoza durante los meses de mayo a agosto. Esta especie representa la mayor abundancia para el estado de Puebla dado a que presenta una amplia distribución. Datos adicionales en Arce-Pérez y Morón (2000) (Figs. 3, 10).

Macrodactylus nigripes Bates, 1887. Los adultos de esta especie se recolectaron en los municipios de Acatzingo, Atlixco, Chinautla, Huauchinango, Hueyapan, San Felipe Teotlancingo, Santiago Zautla, Teziutlán, Tochimilco, Xiutetelco, Zacatlán, Zaragoza, Zacapoaxtla, en los meses de mayo a septiembre. Se observaron daños importantes en el cultivo de $Z$. mays, $P$. vulgaris, $V$. faba y $A$. hypochondriacus, así como a diversos frutales entre los que se encuentran $P$. persica, $P$. comunis, $M$. domestica y $P$. domestica. Por otra parte, se recolectaron larvas asociadas a cultivo de $Z$. mays y A. hypochondriacus. Asimismo, Arce-Pérez y Morón (2000) citan a esta especie en los municipios de Chiautzingo, Chignaguapan, Puebla y San Andrés Cholula. Datos adicionales en Arce-Pérez y Morón (2000) (Figs. 4, 10). 
Macrodactylus rufescens Bates, 1887. Los adultos se habían registrado para los municipios de Teziutlán y Zacatlán (Arce-Pérez \& Morón, 2000), sin embargo, en las recolectas realizadas en este trabajo se registró en los municipios de Atlixco y Tochimilco durante los meses de junio y julio, alimentándose de frutos de E. japonica y de M. domestica. Datos adicionales en Arce-Pérez y Morón (2000) (Figs. 6, 10).

\section{Clave dicotómica para la identificación de las especies del género Macrodactylus presentes en Puebla. \\ Tomada y modificada de Arce-Pérez y Morón (2000).}

1 Pronoto, élitros y placa pigidial glabros. Tegumento elitral notablemente bicolor, contrastante (Grupo Dimidiatus). M. dimidiatus 1' Pronoto, élitros y placa pigidial con vestidura setífera más o menos densa. Tegumento elitral normalmente unicolor, aunque en algunas especies puede tener la mitad posterior obscurecida, pero no contrastante.

2(1') Clípeo aplanado con el ápice claramente truncado y recto; mesotibias con dos espolones rectos; tarsos sin sedas blanquecinas (Grupo Lineatus)................................................................... 3 2' Clípeo aplanado con el ápice truncado y sinuado o ligeramente sinuado o recto; mesotibias con el espolón mayor siempre de ligera a marcadamente recurvado en el ápice; tarsos con anillos de sedas blanquecinas en la base (Grupo Subspinosus).

3(2) Artejos tarsales con sedas espiniformes en toda su superficie; élitros amarillo rojizo, cubiertos por vestidura corta y plana amarilla claro. Parámeros con sedas diminutas en su parte apical.

M. fulvescens

3' Artejos tarsales sin sedas espiniformes en la superficie; élitros homogéneamente coloreados o bicolor. Parámeros glabros.

4(3') Élitros homogéneamente coloreados pardo castaño (ocre); vestidura corta, poco erecta, amarilla clara. Longitud corporal de 9.0 a $10 \mathrm{~mm}$

M. ocreatus

4' Élitros bicolor, la parte anterior amarilla-rojiza y la mitad o tres cuartas partes posterolaterales manchadas de pardo oscuro; vestidura definida por cuatro bandas setíferas amplias de color amarillo claro. Longitud corporal de 10 a $11 \mathrm{~mm}$

M. lineatus

$\mathbf{5 ( 2})$ Superficie dorsal con vestidura de sedas largas, finas y cilíndricas amarillo-blanquecinas que permiten ver el integumento a simple vista.

M. rufescens 5' Superficie dorsal con densa vestidura de sedas largas, gruesas y cilíndricas, de color gris o amarillo, que impide observar el integumento a simple vista.

6(5') Élitros no uniformemente coloreados, con seis estrías desnudas bien marcadas; base de los élitros pardo-rojizo o grisácea, y la mitad posterior obscura. M. mexicanus 6' Élitros uniformemente coloreados, totalmente cubiertos por su vestidura; estrías no visibles o sólo bien marcadas; integumento elitral de color uniforme... 
7(6') Patas negras o pardo oscuro; integumento del pronoto y élitros negro brillante; superficie dorsal con densa vestidura larga, gris o amarilla.

7' Patas nunca negras, excluyendo el ápice de los tarsos y tibias que pueden ser oscuros; vestidura amarillo rojizo o gris cenizo

8(7') Clípeo corto, tan largo como ancho, con sus bordes laterales recurvados y el ápice truncado

M. infuscatus

8' Clípeo ligeramente alargado, con sus bordes laterales rectos, convergentes, y su ápice truncado,

ligeramente sinuado o recto.

$\mathbf{9}\left(\mathbf{8}^{\prime}\right)$ Élitros amarillo-rojizos, con dos bandas anchas de vestidura setífera desde la base hasta el ápice, flanqueadas por otras bandas laterales más angostas. Longitud corporal de 8.5 a $9.4 \mathrm{~mm}$. Parámeros de forma circular.

M. impressus

9' Élitros pardo-amarillentos o rojizos, vestidura dispersa, sin bandas, que permite ver el tegumento; base de los élitros y cerca de la sutura elitral con sedas largas negras evidentes. Longitud corporal de 9 a $10 \mathrm{~mm}$. Parámeros ovalados

M. lineatocollis

AGRADECIMIENTOS. Los autores agradecen al CONACYT el apoyo brindado a través del proyecto "diagnóstico del complejo gallina ciega asociada al cultivo de amaranto en el estado de Puebla" con número de registro CB-2010-01.

\section{LITERATURA CITADA}

Arce-Pérez, R. (2008) Taxonomía y biogeografía de las especies centroamericanas de Macrodactylus Dejean, 1821 (Coleoptera: Melolonthidae). Universidad Autónoma de Hidalgo, Instituto de Ciencias Básicas e Ingeniería, 264 pp.

Arce-Pérez, R., Asiain, J. (2008) Macrodactylus lineatocollis Bates, 1887 (Coleoptera: Melolothidae: Melolonthinae). Dugesiana, 15 (2), 145-146.

Arce-Pérez, R., Morón, M. Á. (2000) Taxonomía y distribución de las especies de Macrodactylus Latreille (Coleoptera: Melolonthidae) en México y Estados Unidos de América. Acta Zoológica Mexicana (nueva serie), 97, 123-239. https://doi.org/10.21829/azm.2000.79791913

Arce-Pérez, R., Morón, M. Á. (2005) New species and new records of species of Macrodactylus Dejean (Coleoptera: Scarabaeidae: Melolonthinae: Macrodactylini) from Honduras and Nicaragua. Zootaxa, 1012, 23-27.

https://doi.org/10.11646/zootaxa.1012.1.3

Arce-Pérez, R., Morón, M. Á. (2009) New species of Macrodactylus Dejean (Coleoptera: Scarabaiedae: Melolonthinae: Macrodactylini) from Mexico. The Coleopterists Bulletin, 63, 501-508. https://doi.org/10.1649/1203.1

Arce-Pérez, R., Morón, M. Á. (2010) Macrodactylus zaragozai sp. nov. (Coleoptera: Scarabaeidae: Melolonthinae: Macrodactylini) from Guatemala and Mexico. Dugesiana, 17, 171-174. https://doi.org/10.32870/dugesiana.v17i2.3959 
Arce-Pérez, R., Morón, M. Á. (2011) Los Macrodactylus (Coleoptera: Melolonthidae) de América Central. Editorial Académica Española, Saarbrücken, Germany, 275 pp.

Arce-Pérez, R., Morón, M. Á. (2014) New species and records of Macrodactylus Dejean (Coleoptera: Scarabaeidae: Melolonthinae: Macrodactylini) from Bolivia. Zootaxa, 3857 (2), 292-300. https://doi.org/10.11646/zootaxa.3857.2.8

Arce-Pérez, R., Morón, M. Á. (2018) Revision of the species of Macrodactylus Dejean (Coleoptera: Scarabaeidae: Melolonthinae: Macrodactylini) from Costa Rica and Panamá. Zootaxa, 4462 (3), 379-403. https://doi.org/10.11646/zootaxa.4462.3.5

Arce-Pérez, R., Morón, M. Á. (2020) Revision of the species of Macrodactylus Dejean (Coleoptera: Scarabaeidae: Melolonthinae: Macrodactylini) from the Central American Nucleus. Zootaxa, 4772 (3), 567-584. https://doi.org/10.11646/zootaxa.4772.3.7

CIBCEC (2003) Dirección General Adjunta de Planeación Microrregional, Unidad de Microregiones. Disponible en: http://www.microrregiones.gob.mx/cedulas/localidadesDin/ubicacion/relieve.asp?micro= SIERRA\%20NEGRA\&clave $=212090011 \&$ nomloc $=$ SAN\%20PEDRO\%20ATZUMBA (consultado 12 enero de 2021).

Coto, D., Saunders J. L. (2004) Insectos plagas de los cultivos perennes con énfasis en frutales en América Central. Centro Agronómico Tropical de Investigación y Enseñanza. Turrialba, Costa Rica, 118 pp.

Evans, A. V. (2003) Checklist of the New World chafers (Coleoptera: Scarabaeidae: Melolonthinae). A checklist of the New World chafers (Coleoptera: Scarabaeidae: Melolonthinae). Zootaxa, 211, 1-458.

Evans, A. V., Smith, A. B. T. (2005) An electronic checklist of the New World chafers (Coleoptera: Scarabaeidae: Melolonthinae). Version 3. Electronically published, Ottawa, Canadá, 353 pp. https://doi.org/10.11646/zootaxa.211.1.1

García, E. (1990) Rangos de humedad. Extraído de Climas. IV.4.10. Atlas Nacional de México. Vol II. Escala 1: 4000000. Instituto de Geografía UNAM. México.

Téllez-Carmona, J. M., Aragón-García, A., Pérez-Torrez, B. C., Nava-Díaz, C., MolinaMartínez, A. (2018) Entomofauna asociada al cultivo de chía (Salvia hispanica L.) en San Lucas Tulcingo, Tochimilco, Puebla. México. Entomología Mexicana, 5, 358-361.

Saunders, J. L., King, A. B. S., Vargas, C. S. (1983) Plagas de cultivos en América Central una lista de referencia. Centro Agronómico Tropical de Investigación y Enseñanza. Turrialba, Costa Rica, $91 \mathrm{pp}$. 